\title{
Some remarks on Oscillating Inflation
}

\author{
V. Cárdenas* and G. Palma ${ }^{\dagger}$ \\ Departamento de Física, Universidad de Santiago \\ Casilla 30\%, Correo 2, Santiago, Chile.
}

(February 7, 2020)

\begin{abstract}
In a recent paper Damour and Mukhanov describe a scenario where inflation may continue during the oscillatory phase. This effect is possible because the scalar field spends a significant fraction of each period $f$ oscillation on the upper part of the potential. Such additional period of inflation could push perturbations after the slow roll regime to observable scales. Although we show that the small region of the Damour-Mukhanov parameter q gives the main contribution to oscillating inflation, it remained until now not satisfactory understood. Further, it drives to an expression for the energy density spectrum of perturbations, which is well behaved in the whole physical range of $\mathrm{q}$.
\end{abstract}

PACS numbers: 98.80.Cq

*E-mail: vcardena@lauca.usach.cl

†E-mail: gpalma@lauca.usach.cl 


\section{INTRODUCTION}

Nowadays inflation is a widely accepted element of the early cosmology [1]. It gives the possibility of solving many of the shortcomings of the standard hot big bang model and provides the source for the early energy density fluctuations responsible of the large scale structure of the universe observed today. Although there are many models of inflation, the underlying physical ideas are well established. These are characterized by a period of "slow roll" evolution of a scalar field (inflaton) toward the vacuum potential. During this period the field changes very slowly, so that the kinetic energy $\dot{\varphi}^{2} / 2$ remains smaller than its potential energy $V(\varphi)$ and the energy density associated to the scalar field acts as a "cosmological constant" term allowing a period of quasi exponential expansion of the scale factor. When the period of inflation ends, the scalar field $\varphi$ start a phase of rapid coherent oscillations around the vacuum.

Very recently [2,3] it has been stressed that inflation can persist during the coherent oscillations of the inflation field phase. This exciting result is possible when the inflaton potential verifies a simple constrain of curvature far from the core convex part, where the inflaton can roll slowly. The efficiency of this phenomena could have important implications for GUT scale baryogenesis. In fact, as was suggested by Damour and Mukhanov, it could be expected that due to the increase of the oscillation frequency, there is the possibility to generate massive particles heavier than $\sim 10^{16} \mathrm{Gev}$

[4]. In [2] Damour and Mukhanov estimated the amount of inflation to be $\sim 10$ efold (powers of the scale factor). They argue that this effect can be more efficient than the parametric resonance proposed by Kofman, Linde and Starobinsky [5]. In [3] Mazumdar and Liddle showed that Mukhanov et.al. overestimated the number of e-fold because they have used a slow-roll definition of this object. In their paper, Mazumdar et.al. found an analytical expression for the number of e-fold using the appropriate definition finding a number of $\sim 3$ e-fold concluding that this effect is not very efficient. The study of adiabatic perturbations in this phase has been made by Taruya [6]. He found a poor amplification in the case of a single scalar field model but anticipated an enormous amplification for multi-field systems.

In this letter we revisit the subject. In particular we find that the analytical expressions used to compared with the numerical estimation are not well defined in the $q \sim 0$ region and propose a way to correct these analytical estimations. Further, with this result we study the evolution of the scalar field finding total agreement with the conclusions of [3] for $q>0$, but an exciting different result for small $q$. For this region, the initial conditions are very important. We find that $q \sim 0$ gives the leading contribution for oscillating inflation and the dominant part in the amplification of the fluctuations.

The letter is organized as follow; first we describe the Damour-Mukhanov model. Then, we made some comments about initial conditions for this phenomenon and later propose our contribution. 


\section{BASIC EQUATIONS}

Now we shall restrict ourselves to models of inflation driven by a single scalar field. The equations are

$$
\begin{aligned}
& \ddot{\varphi}+3 H \dot{\varphi}+V,_{\varphi}=0, \\
& H^{2}=\kappa^{2}\left(\frac{1}{2} \dot{\varphi}^{2}+V\right),
\end{aligned}
$$

Here $H=\dot{a} / a$ is the Hubble parameter, $a$ is the scale factor of the universe and $\kappa^{2}=8 \pi / 3 M_{p}^{2}$ with $M_{p}=1.2 \cdot 10^{19}$ Gev the Planck mass. During the oscillatory phase of $\varphi$ we have two time scales; the frequency $\omega$ of $\varphi$ and the rate of expansion $H$. If the limit $\omega \gg H$ is taken we can neglect terms proportional to $H$ in the equations. So from (1) we can integrate to obtain

$$
\rho=\frac{1}{2} \dot{\varphi}^{2}+V=c t e=V_{m}
$$

where $V_{m}=V\left(\varphi_{m}\right)$ is the maximum value of $V(\varphi)$ in each oscillation when the field reaches the maximum value $\varphi_{m}$. From this relation we obtain the period of a single oscillation,

$$
T=4 \int_{0}^{\varphi_{m}} d \varphi\left[2\left(V_{m}-V(\varphi)\right]^{\frac{1}{2}} .\right.
$$

When $\omega \ll H$ we can define an adiabatic average index $\gamma$ by:

$$
\gamma=\left\langle\frac{\rho+p}{\rho}\right\rangle
$$

where the bracket means $\langle\ldots\rangle=T^{-1} \int_{0}^{T} \ldots d t$. Rewritten the equations $(1,2)$ in the fluid form

$$
\begin{aligned}
& \dot{\rho}=-3 H(p+\rho), \\
& \frac{\ddot{a}}{a}=-\frac{1}{3}(\rho+3 p),
\end{aligned}
$$

then from (4) and (5, 6) we have several ways to compute the adiabatic index

$$
\gamma=\frac{\left\langle\dot{\varphi}^{2}\right\rangle}{V_{m}}=\frac{\left\langle\varphi V_{, \varphi}\right\rangle}{V_{m}}=2\left(1-\frac{\langle V\rangle}{V_{m}}\right)
$$

Because $p=(\gamma-1) \rho$ and (6) we have a superluminal expansion $\ddot{a}>0$ when $\gamma<2 / 3$. From the last two relations in (7) the inequality $\gamma<2 / 3$ leads to

$$
\left\langle V-\varphi V_{, \varphi}\right\rangle>0 .
$$




\section{DAMOUR-MUKHANOV MODEL}

Until now everything has been done for an arbitrary potential, but from now on we shall consider the potential

$$
V(\varphi)=\frac{A}{q}\left[\left(\frac{\varphi^{2}}{\varphi_{c}^{2}}+1\right)^{q / 2}-1\right]
$$

where $q$ is a dimensionless parameter, $A=[\text { mass }]^{4}$ is a constant and $\varphi_{c}=[$ mass $]$ determinies the size of the convex core of $V(\varphi)$. We assume for a while that $\varphi_{c}$ marks the end of oscillating inflation. The analysis made in [2] works well far from the core of the potential. Further, the limit $\varphi \gg \varphi_{c}$ of (9) was written as

$$
V(\varphi) \simeq \frac{A}{q}\left(\frac{\varphi}{\varphi_{c}}\right)^{q} .
$$

In this case, the adiabatic index can be computed exactly [7] and is given by

$$
\gamma=\frac{2 q}{q+2}
$$

so, from the inequality $\gamma<2 / 3$ we note that to hold inflation during the oscillatory phase we must have $q<1$. Another way to obtain this result is to link the inflexion point $\tilde{\varphi}$ of $V(\varphi)$ and $\varphi_{c}$. From (9) we obtain

$$
\tilde{\varphi}^{2}=\frac{\varphi_{c}^{2}}{1-q}
$$

then, to have a real $\tilde{\varphi}$ we must impose $q<1$, that is, the condition for inflation to hold. It suggest us that in general the final field configuration depends on the parameters of the potential as we will see later. By using (11) in eq.(5) we obtain $\dot{\rho}=-3 H \gamma \rho$ and together with eq.(2) we have [2]

$$
\begin{gathered}
a \propto t^{2 / 3 \gamma}=t^{(q+2) / 3 q}, \\
\varphi_{m} \propto t^{-2 / q} \propto a^{-6 /(q+2)}, \\
\rho=V\left(\varphi_{m}\right) \propto t^{-2} \propto a^{-6 q /(q+2)},
\end{gathered}
$$

where $\varphi_{m}$ is the amplitude of the oscillations, $\varphi_{c}<\varphi_{m}<\varphi_{s}$ and $\varphi_{s}$ is a typical value of $\varphi$ at the end of inflation, and the beginning of oscillating inflation. To compute the number

of e-fold of inflation during oscillating inflation we can not use the standard expression $N=\ln a_{f} / a_{i}$, appropriate for the slow-roll stage, but the improved expression proposed by [3]

$$
\tilde{N}=\ln \frac{a_{f} H_{f}}{a_{i} H_{i}}
$$


because in each oscillation, while the field spends time in the core region, the universe continue their expansion so $H$ can vary. Then from (2) and (14) $H \propto a^{-3 q /(q+2)}$ the product $a H \propto \varphi_{m}^{(1-q) / 3}$ and from (15) we obtain

$$
\tilde{N} \simeq \frac{1-q}{3}\left[\ln \frac{q M_{p}}{\varphi_{c}}-2\right],
$$

where we have used $\varphi_{s} \sim q M_{p} / \sqrt{16} \pi$. In [3] the numerical curves for $\varphi_{c}=10^{-6} M_{p}$ show that $\tilde{N} \lesssim 3$. Using the analytical expression (16) we do not find agreement for small values of $q$. However there is not a compelling reason to believe in (16) for small $q$.

\section{THE SMALL Q-REGION}

To study the small $q$ region, we must use the correct limit $q \rightarrow 0$ of (9) which leads to

$$
V(\varphi) \simeq \frac{A}{2} \ln \left[\left(\frac{\varphi}{\varphi_{c}}\right)^{2}+1\right],
$$

so, if now we take the limit $\varphi \gg \varphi_{c}$ we obtain the logarithmic potential $V(\varphi) \simeq A \ln \left(\varphi / \varphi_{c}\right)$. A very important fact to note from (10) is that the limit $q \rightarrow 0$ does not exist. Of course, the expression (10) is wrong around the $q \sim 0$ region and the expressions derived from this are ill-defined. But, some work has been done in this regard [2]. For the logarithmic potential the adiabatic index is $\gamma=1 / \ln \left(\varphi_{m} / \varphi_{c}\right)$, so from (5) and (2) we obtain

$$
a(t) \propto \exp \left[-\frac{A}{2}\left(t_{\text {end }}-t\right)^{2}\right],
$$

but this form does not permit us to write explicit expressions for $\tilde{N}$ (see [3]). Let us make some comments about this result. Because $\gamma=1 / \ln \left(\varphi_{m} / \varphi_{c}\right)$ from (5) we obtain $\dot{\rho}=-3 H A$, then $a \sim\left(\varphi_{m} / \varphi_{c}\right)^{-1 / 3}$. Moreover, from (2) we have $H \propto \rho^{1 / 2} \propto\left(\ln \left(\varphi_{m} / \varphi_{c}\right)\right)^{1 / 2}$, then to compute $\tilde{N}$ we must evaluate the factor $\left(\ln \left(\varphi_{m} / \varphi_{c}\right)\right)^{1 / 2}\left(\varphi_{m} / \varphi_{c}\right)^{-1 / 3}$ at the extremes $\varphi_{s}$ and $\varphi_{c}$, but this is not be possible. The problem arises when we put $\varphi_{m}$ close to $\varphi_{c}$ in a expression valid for $\varphi \gg \varphi_{c}$. Because (10) is valid for $\varphi \gg \varphi_{c}$ too, we expect to see the same behavior in the calculation of $\tilde{N}$. In fact this is the case but, to see that, we must include the constant term $A / q$ in (10) or equivalently, write the limit correctly.

When we use the potential

$$
V(\varphi) \simeq \frac{A}{q}\left[\left(\frac{\varphi}{\varphi_{c}}\right)^{q}-1\right],
$$

the adiabatic index become time-dependent, satisfying the equation

$$
\gamma \rho=\frac{2 q}{q+2}\left(\rho+\frac{A}{q}\right),
$$

where $\rho(t)=V\left(\varphi_{m}(t)\right)=A\left[\left(\varphi_{m}(t) / \varphi_{c}\right)^{2}-1\right] / q$. Replacing this in (5) we obtain the same behavior as in eq.(13). But when we calculate $H$ we obtain $H \propto \rho^{1 / 2} \propto\left[\left(\varphi_{m} / \varphi_{c}\right)^{2}-1\right]^{1 / 2}$, and the point $\varphi_{m}=\varphi_{c}$ is not allowed, finding the same behavior mentioned before. 
To avoid this, we investigated other way to compute $H$ valid near the core region. This procedure is valid because we are interested just in relative weights of $H$ and not in its exact value. An appropriate expression to be use here is (9) and we obtain $\tilde{N} \simeq 2.6$ for $\varphi_{c}=10^{-6} M_{p}$ and $q=0.1$ and $\tilde{N} \simeq 2.75$ for $q=0.01$. Further, the limit $q \rightarrow 0$ exists leading to $\tilde{N}=2.76(2)$, because we can made this calculation using the limit $q \rightarrow 0$ in (9) i.e., the logarithmic form (17), in this way we show that this limit exists. It should be noted that this results must be compared with equation (17) of reference [3]. The next step is find a expression for $\varphi_{s}$ in the model under consideration.

The authors of [3] found $\varphi_{s} \sim q M_{p} / \sqrt{16} \pi$ using the potential (10). From this result they found a decrease of $N$ at decreasing values of $q$. This seems very strange because, for smaller values of $q$ we obtain flatter potentials at $\varphi \gg \varphi_{c}$, then we will have more time for inflation. Furthermore, we are not safe of how they set the initial conditions for $\varphi$ in their numerical analysis. In general, the initial and final field configuration depends on the potential.

For $q$ near cero, $\varphi_{s}$ is not proportional to $q$. In fact, we know that $\varphi_{s}$ came from the saturation of the slow-roll inequality $\left|V^{\prime} / V\right|<\sqrt{48 \pi} / M_{p}$ but for $q \sim 0$ the potential is (18) and we obtain for $\varphi_{s}$ the equation

$$
\left(\frac{\varphi_{s}}{\varphi_{c}}\right)^{q-1}=\frac{\sqrt{48 \pi} \varphi_{c}}{q M_{p}}\left[\left(\frac{\varphi_{s}}{\varphi_{c}}\right)^{q}-1\right] .
$$

Near $q \sim 0$ we have for $\varphi_{s}$

$$
\varphi_{s} \ln \frac{\varphi_{s}}{\varphi_{c}}=\frac{M_{p}}{\sqrt{48 \pi}},
$$

and there is no dependence on $q$. In Fig. 1 we see the behavior of $\varphi_{s}$ in terms of $q$ given by (19). Of course, for $q>0$ both curves agree. Nevertheless when $q \rightarrow 0$ the field $\varphi_{s}$ grows preventing the fall off $\tilde{N}$ predicted in [3] (see Fig. 2).

Moreover, as we have anticipated before, the value of the field at the end of oscillating inflation will have a $q$-dependence too. We know from [2] that the intercept $U(\varphi)=V(\varphi)-$ $\varphi V_{, \varphi}$, must be positive to hold oscillating inflation. Let us define $\varphi_{f}$ to be the value of the inflaton field $\varphi$ at which $U\left(\varphi_{f}\right)=0$. This condition represents the end of inflation due to oscillation. We need thus to compare $\varphi_{f}$ for different values of $q$.

If we take the potential (18) and define $x=\varphi / \varphi_{c}$, we obtain

$$
U(x)=\frac{A}{q}\left[x^{q}(1-q)-1\right] .
$$

From this equation we can extract a explicit expression for $\varphi_{f}$. If we impose $U\left(x_{f}\right)=0$ we obtain the value of the scalar inflaton field at the end of this phase

$$
\varphi_{f}=\varphi_{c}(1-q)^{-1 / q} .
$$

From (15) and (16) the correct e-fold number is $\tilde{N} \simeq(1-q) \ln \left(\varphi_{i} / \varphi_{f}\right) / 3$, where $\varphi_{i}=\varphi_{s}$ is the value of the scalar field at the beginning of oscillating inflation phase. Then we obtain

$$
\tilde{N} \simeq \frac{1-q}{3}\left[\ln \frac{q M_{p}}{\varphi_{c}}-2+\frac{\ln (1-q)}{q}\right] .
$$


The last contribution in the square brackets leads to a still smaller amount of inflation. In figure 3, we show the combined result of both effects. Because $\varphi_{f}$ is greater than $\varphi_{c}$ the amount of inflation is small in the whole range of $q \in(0,1)$. Moreover, the corrected values of $\varphi_{s}$ given by (19) keep $\tilde{N}$ to be a decreasing function of $q$ even in the small $q$-range.

\section{DENSITY PERTURBATIONS}

Because oscillating inflation add e-folds of inflation after the slow-roll regime where the observed perturbations are generated, is possible that this additional period of inflation could push the perturbations to observable scales. In order to obtain the required amplitude of density perturbations and does not give an unphysical constraint on the potential, we must compute the density perturbation spectrum for the model being studied and then compare. In reference [3] an expression for this object was derived

$$
\delta_{H}^{2}=\frac{512 \pi}{75} \frac{A}{q^{3} M_{p}^{6}} \frac{\varphi^{2+q}}{\varphi_{c}^{q}} .
$$

However, for $q \sim 0$ this expression is not well defined. Using the primordial density perturbation spectrum $\delta_{H}$ as was define in [3] we obtain for (18)

$$
\delta_{H}^{2}=\frac{512 \pi}{75} \frac{A}{q^{3} M_{p}^{6}}\left\{\frac{\left[\left(\varphi / \varphi_{c}\right)^{q}-1\right]^{3}}{\left(\varphi / \varphi_{c}\right)^{2 q}}\right\},
$$

who is well define when we take the $q \rightarrow 0$ limit. In this limit it take the form

$$
\delta_{H}^{2}=\frac{512 \pi}{75} \frac{A}{q^{3} M_{p}^{6}} \ln ^{3}\left(\frac{\varphi}{\varphi_{c}}\right) .
$$

Because the COBE satellite require $\delta_{H} \approx 2 \cdot 10^{-5}$, in the $q=0$ case the amplitude of the potential for $\varphi_{c}=10^{-6} M_{p}$ gives $A^{1 / 4} \sim 2 \cdot 10^{-3} M_{p}$, which is a typical number for inflationary models.

\section{SUMMARY}

We have made some corrections about how compute the number of e-fold, which account the amount of inflation during the oscillatory phase. In particular we note that previous studies are not accurate because they are not valid close to the core of the potential $\varphi \sim \varphi_{c}$. Thus, we make the analysis for the small $q$ region of the potential, which has not been considered until now. Finally we find, in order to extract the correct amount of inflation during this phase, that a very careful definition of initial and final field configuration are needed. Our results show that near $q \sim 0$ the e-fold number is maximal but it is not still enough to be more efficient than the parametric resonant effect discussed in [5]. 


\section{ACKNOWLEDGMENTS}

The authors want to thank to Sergio del Campo for a critical reading of the manuscript. V. Cárdenas wants to thanks CONICYT for support through a scholarship. G. Palma was supported in part through Proyect FONDECYT 1980608 and Proyect DICYT 049631PA. 


\section{REFERENCES}

[1] A. H. Guth, Phys. Rev. D 23, 347 (1981); A. Linde, Particle Physics and Inflationary Cosmology, Harwood NY (1990); E. Kolb and M. Turner, The early Universe, AddisonWesley (1990).

[2] T. Damour and V. Mukhanov, Phys. Rev. Lett. 80, 3440 (1998), gr-qc/9712061.

[3] A. Mazumdar and A. Liddle, Phys. Rev. D 58, 083508 (1998), astro-ph/9806127.

[4] E. Kolb, A. Linde and A. Riotto, Phys. Rev. Lett. 77, 4290 (1996).

[5] L. Kofman, A. Linde and A. Starobinsky, Phys. Rev. Lett. 73, 3195 (1994): Phys. Rev. Lett. 76, 1011 (1996): Phys. Rev. D 56, 3258 (1997).

[6] A. Taruya, astro-ph/9812342.

[7] M. Turner, Phys. Rev. D 28, 1243 (1983). 


\section{FIGURES CAPTION}

Figure 1: We plot the q dependence of $\varphi_{s}$. We see that for $q>0$ both curves agree but for $q \sim 0$ the field grows preventing the fall of $\tilde{N}$.

Figure 2: $\quad$ The e-fold number $\tilde{N}$ as a function of $q$ taking into account the behavior of $\varphi_{s}$ described for the Eq. (19).

Figure 3: $\quad$ The e-fold number $\tilde{N}$ versus $q$ taking into account both effects: the behavior of $\varphi_{s}$ described by eq. (19) and the definition of $\varphi_{f}$ discussed in the text. 


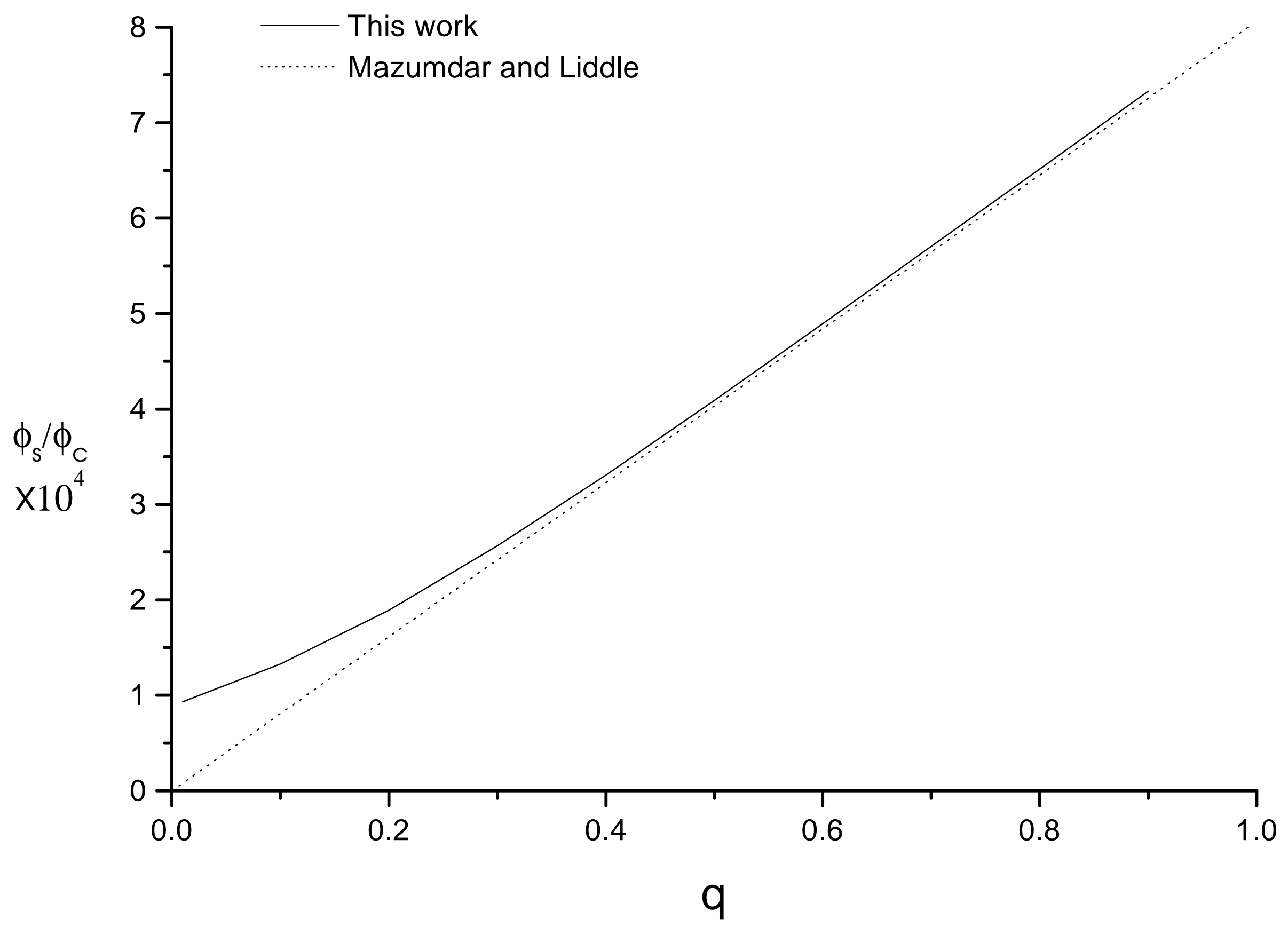




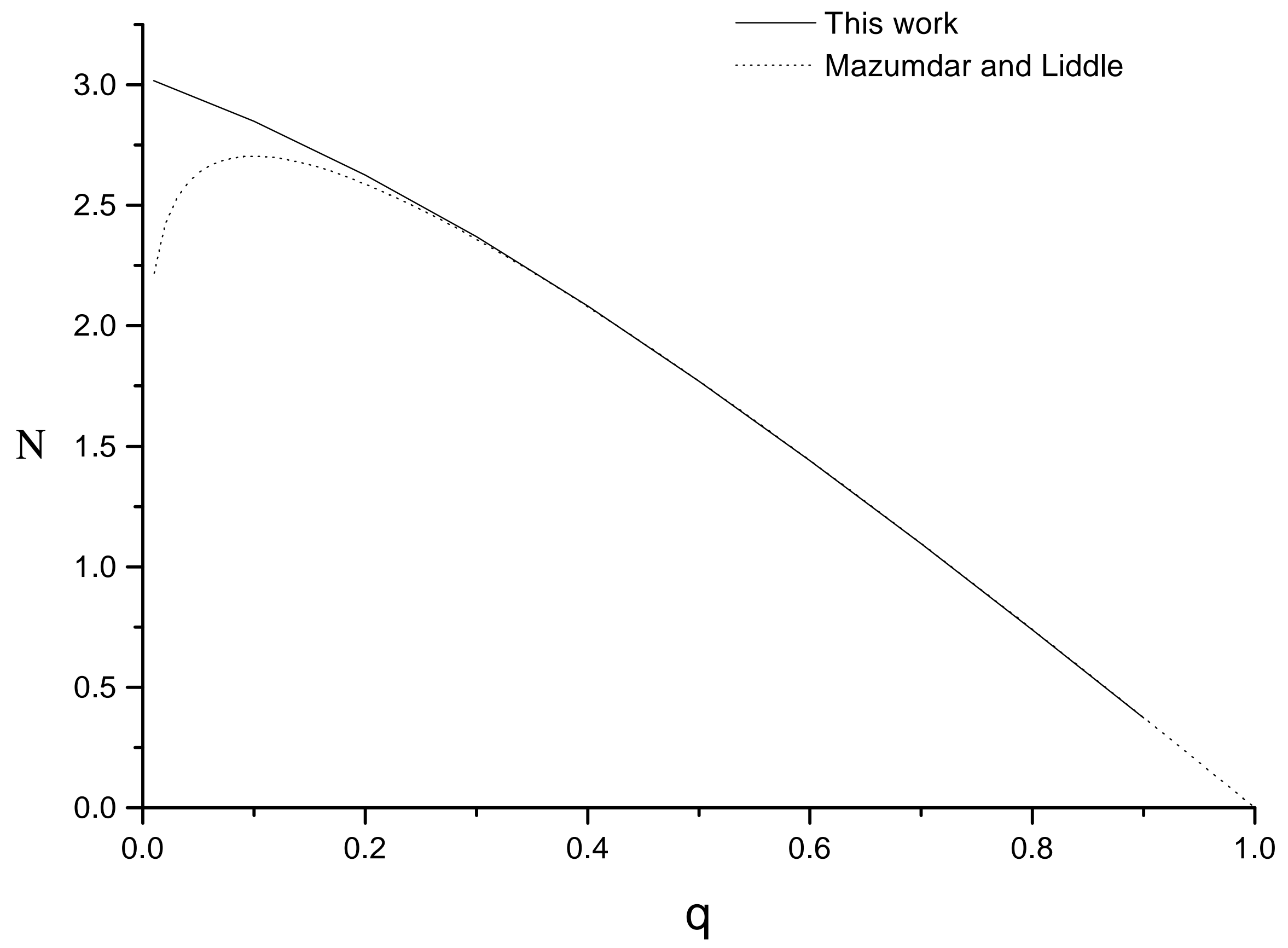




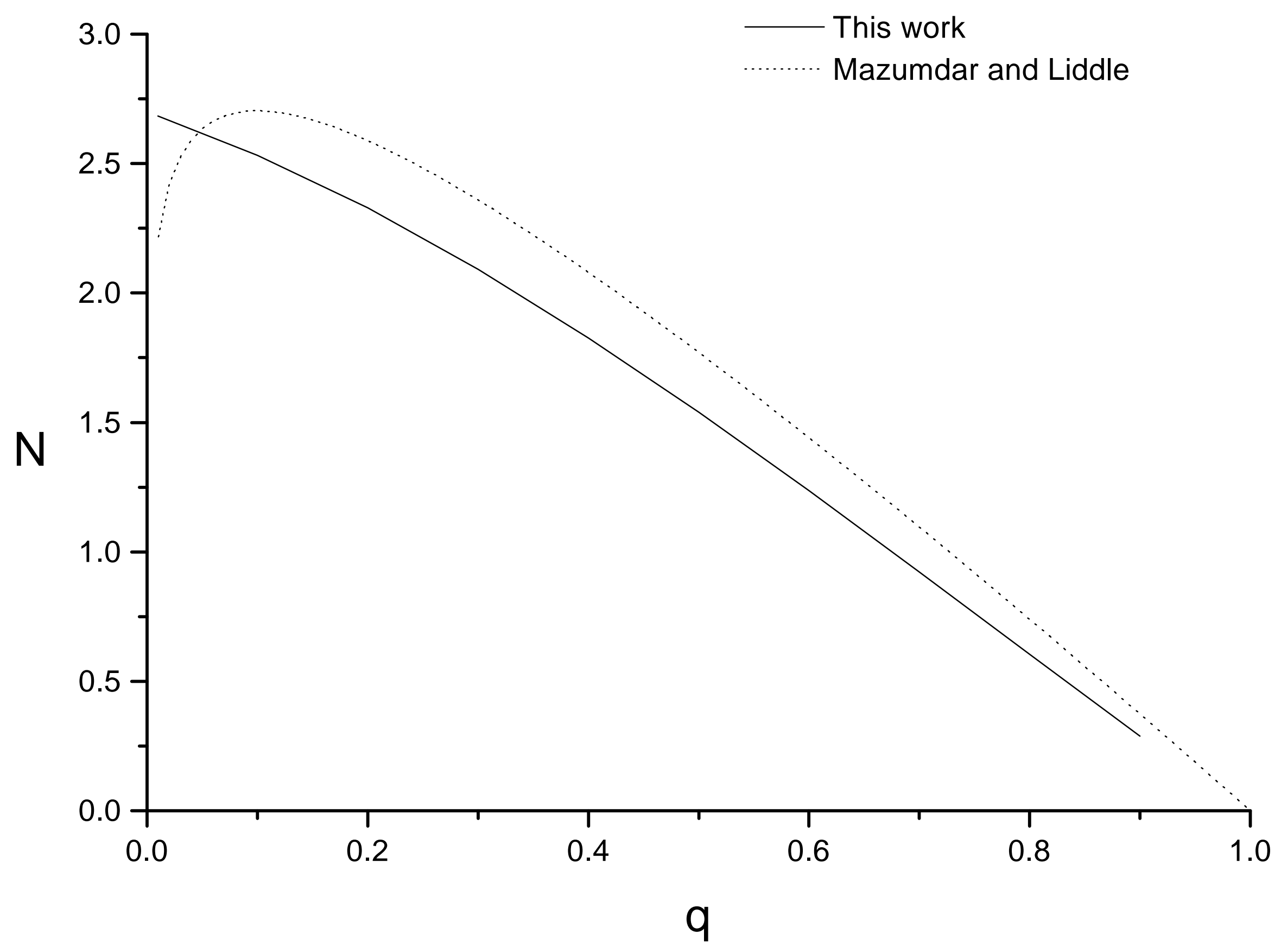

